\title{
Value-at-Risk Estimation of Equity Market Risk in India
}

\author{
Jitender \\ School of Economics, University of Hyderabad, Hyderabad-500046 (India) \\ jitendergame@gmail.com
}

\begin{abstract}
The value-at-risk (Va) method in market risk management is becoming a benchmark for measuring "market risk" for any financial instrument. The present study aims at examining which VaR model best describes the risk arising out of the Indian equity market (Bombay Stock Exchange (BSE) Sensex). Using data from 2006 to 2015, the VaR figures associated with parametric (variance-covariance, Exponentially Weighted Moving Average, Generalized Autoregressive Conditional Heteroskedasticity) and non-parametric (historical simulation and Monte Carlo simulation) methods have been calculated. The study concludes that VaR models based on the assumption of normality underestimate the risk when returns are non-normally distributed. Models that capture fat-tailed behaviour of financial returns (historical simulation) are better able to capture the risk arising out of the financial instrument.
\end{abstract}

Keywords: value-at-risk (VaR), equity market risk, variance-covariance, historical simulation, financial risk management

JEL Classification: C52, C53

\section{Introduction}

The research on financial risk management in India has from a long time been concentrated on mainly credit risk. The extent of risk posed by market risk instruments in Indian financial market has not been systematically and deeply studied. There is a relatively less number of studies dealing with market risk as compared to credit risk. This is largely because Indian financial market has from a long time been dominated by mainly credit products relating to retail, auto, housing, and personal finance. The commercial banks in India have traditionally focused mainly on the borrowing and lending business, which primarily generates credit risk. As the world is becoming more and more integrated in financial flows and the Indian financial market is also becoming more and more open to foreign 
portfolio investments, there is a need for a systematic study to quantify the market risk from equity market and develop the best predictable statistical model which can be leveraged for forecasting purposes.

The present paper is trying to bridge this gap by studying the predictive accuracy of various VaR models for the Indian equity market (Sensex). This study can help market participants, traders, risk managers, and researchers who are interested in systematic and quantitative study on the Indian financial market. Sensex is taken as a benchmark index to represent the Indian equity market as it is considered the barometer of Indian equity market. Sensex is a composite index of the 30 most actively traded stocks in India. It is calculated using free-float methodology. Free-float methodology market capitalization is calculated by taking the current market share price and multiplying it by the number of shares available in the market for trading.

Value at risk (VaR) is a statistical concept and is itself just a number, used mainly to calculate the market risk of a financial instrument. It is the maximum loss that an institution can be confident it would lose on a financial instrument with a given level of confidence interval due to "normal market" movements within a specified period of time. Losses greater than VaR are supposed to occur with an already specified probability, which is known as the level of significance, or "Type I error" in statistics (Figure 1).

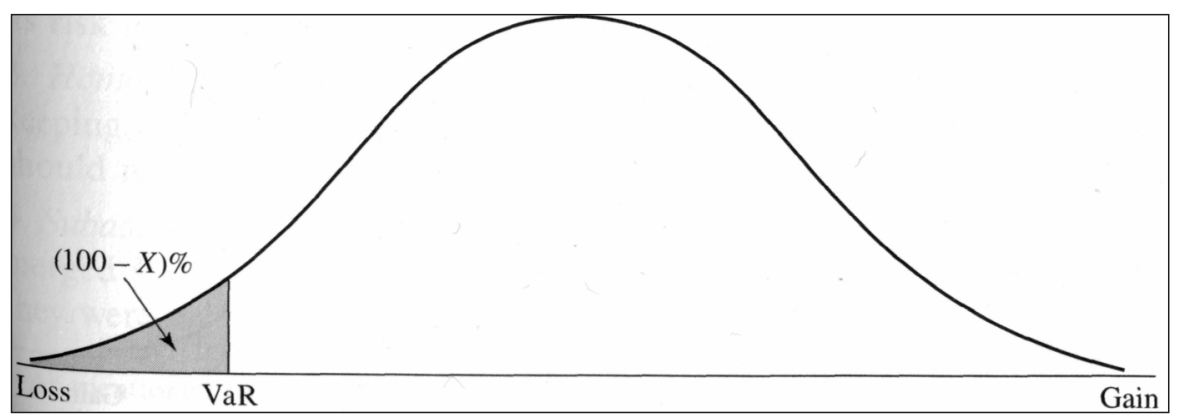

Figure 1. $V a R$

Figure 1 above shows that VaR is calculated from the left tail of the return distribution. VaR is concerned with forecasting the losses with the desired confidence level $(x \%)$ from the return distribution.

The rest of the study is organized as follows. In Section 2, a brief review of highly relevant literature on VaR is provided. Section 3 describes the methodology of the present study and discusses the methods used for VaR calculation. Section 4 provides the empirical results obtained. Section 5 provides the backtesting results. Finally, Section 6 provides the summary and conclusions of the study. 


\section{Literature Review}

The use of normal distribution of asset returns is found to be inappropriate by many empirical studies. Venkatraman (1997) recommends a mixture of various distributions as an alternative to normal distribution. The parameters of the mixture of normal distributions are examined by three methodologies: traditional maximum likelihood, the quasi-Bayesian maximum likelihood, and the Bayesian approach proposed by Zangari (1996). The performance of this method is analysed using the foreign exchange data for eight currencies over the period from 1978 to 1996. The results indicate that the mixture of normal distributions shows smaller errors than the normal approach at high confidence intervals. He also found that normal distribution approach underestimates VaR at very high levels of confidence and overestimates it at lower confidence levels.

Varma (1999) carries out an empirical analysis of VaR models for the Indian stock market. The results indicate that VaR model estimates are critically dependent upon the estimates of the volatility of the underlying asset and that exponentially weighted moving average (EWMA) models do well at higher risk levels, namely at $10 \%$ or $5 \%$, but break down at $1 \%$.

Maria Coronado (2000) compared the results of historical simulation, variancecovariance methods, and the Monte Carlo simulation method as a measurement of market risk for actual nonlinear portfolios in the context of the supervision of bank solvency. In the results, she found that VaR estimates differ significantly across the methods, and the difference is even higher if the confidence level is higher.

Nath and Samanta (2003) calculate the VaR figures and corresponding capital charges for two portfolios with different VaR models such as variance-covariance approach, historical simulation, and tail-based approach. Results show that the variance-covariance approach in the form of risk metrics database underestimates VaR figures, whereas the historical simulation approach provides quite reasonable VaR estimates.

Aktham I. \& Haitham (2006) compare the performance of VaR models for seven Middle Eastern and North African (MENA) countries. Results demonstrate that the extreme value theory approach provides accurate VaR estimates. This implies that the MENA market returns are characterized by fat tails.

Harmantzis et al. (2006) analyse VaR for the daily returns of six equity indices and four currency pairs for a period covering 10 years. They considered various equity indices and currency pairs such as S\&P500, DAX, US dollar vs. euro, or US dollar vs. yen. Results show that the historical simulation model produces accurate forecasts compared to models based on normal distribution approach.

Kisacik (2006) examines the performance of VaR methods in the presence of high volatility and heavy tails. He categorizes VaR methods into traditional and alternative approaches, where the later includes the extreme value theory 
approach. He observes the existence of non-normal distribution. From the empirical comparison of VaR methods, he observes that generalized Pareto distribution works well in the tail of distribution, that is, where the quantile is $99 \%$ or more. Traditional methods provide good results in lower quantiles, that is, less than $99 \%$. Thus, in the presence of high volatility and heavy tails, EVT provides better results than traditional methods for examining tail events.

Dutta and Bhattacharya (2008) show that bootstrapped historical simulation VaR is a better technique than the ordinary historical simulation approach as it keeps the true distributional property along with tackling the scarcity of adequate data points. They calculate VaR and expected shortfall with the historical simulation method and bootstrapped historical simulation method by taking the S\&P CNX Nifty data over the period of 1 April 2000-31 March 2007. They use 95\% confidence level with a five-day time horizon. From the graphical plot of profit and loss of index return and Quantile-Quantile (QQ) plot, they show that the assumption of normal distribution is not appropriate for the return distribution. In their VaR estimation, they find that the bootstrapped historical simulation VaR figure is lower than the ordinary historical simulation estimate.

Sollis (2009) evaluates various VaR approaches under the Basel II regulatory framework. The author mentions that there are flaws in the VaR models which failed to predict the global financial crisis of 2008. The author criticizes the variancecovariance model developed by RiskMetrics. He mentions that variance-covariance is used by many financial institutions because of its simplicity in understanding and calculation. The variance-covariance model assumes that assets are normally distributed. The author observes that assets' returns do not follow the normal distribution. As such, the VaR estimates based on the variance-covariance approach will underestimate true VaR.

Samanta et al. (2010) study the market risk of selected government bonds using VaR models in India. The authors employ distributions based on non-normal assumptions about asset returns such as historical simulation and the extreme value theory. The authors empirically observe that the historical simulation method is able to provide accurate VaR numbers for the Indian Bond Market.

Emrah, Sayad, and Levent (2012) compared and ranked the predictive ability of different VaR models. They argue that the credit crisis period (2007-2009) offers an exclusive opportunity for analysing the success of different VaR models both in developing and developed countries. They propose a VaR ranking model which tries to minimize the magnitude of errors between predicted losses and actual losses, also reducing the autocorrelation problems of the residuals. Results demonstrate that EGARCH gives better VaR estimates. Their results also indicate that the performance of different VaR models depends on how effectively the asymmetric behaviour is captured by the VaR models and not so much on whether they belong to parametric, non-parametric, semi-parametric, or hybrid models. 
Chowdhury \& Bhattacharya (2015) studied the appropriate VaR method following the global financial crisis by focusing on major Indian sectors listed on the National Stock Exchange. They created the hypothetical portfolio with selected sectorial indices and used VaR to estimate the portfolio risk. They conclude that the Monte Carlo simulation method provides the most appropriate results.

Poornima \& Reddy (2017) compared the market risk of domestic and international hypothetical portfolios using the VaR-CoVaR (variance-covariance) model. They used daily closing prices from 2000 to 2014 of Nifty Spot (NSR), Nifty Future (NFR), INRUSD currency pair Spot (USR), and INRUSD currency pair Future (UFR) for constructing a hypothetical domestic portfolio. Data from January 2000 to December 2014 of BRICS nations, US, and UK equity market indices are taken for constructing an international portfolio. They find that the VaR-CoVaR model provides accurate results at $95 \%$ and $90 \%$ confidence intervals.

Overall, it has been observed empirically by many studies that financial asset returns are very often non-normally distributed, characterized by heavy tails, and in need of being incorporated by using appropriate statistical methods which can incorporate this non-normality and heavy-tailed behaviour.

\section{Methodology of the Study}

The various methodologies of $\mathrm{VaR}$ can be divided into two broader categories. The first one includes the "parametric methods" of VaR (variance-covariance $(\mathrm{VcV})$, exponentially weighted moving average (EWMA), and generalized autoregressive conditional heteroskedasticity (GARCH). These methods mainly involve the estimation of parameters (volatility) of the assumed theoretical probability distribution function. All three parametric methods use different ways of estimating volatility but assume the underlying return distribution to be normal. The second category is based on simulation methods. In this category, the two major model methodologies are the historical simulation (HS) and Monte Carlo simulation (MCS) methods. These methods do not involve any estimation of the parameters of the distribution. They use simulations to generate the return distribution.

\subsection{The Variance-Covariance Method}

In this method of calculating VaR, the main focus is on the volatility of the underlying asset's return. It is a parametric method and assumes that multiple asset returns are multivariate normally distributed. The most important input in parametric VaR is the volatility of past return series. Various methods are there to estimate the volatility, and most of them require analysing past data series. Standard deviation is the simplest and most widely used method of calculating volatility 
from past data and is used in the present study for generating VaR estimations based on the variance-covariance method. This method uses data on volatilities and correlations of various assets, and then it calculates VaR by applying weights to the positions. It involves the following steps: (a) mark-to-market the current portfolio, (b) set the time horizon, (c) set the confidence level, and (d) draw the VaR figure.

This method calculates the 1-day VaR with 99\% confidence level as:

$$
\text { VcV VaR = Amount of the position } \times 2.33 \sigma_{t} \text {, }
$$

where $\sigma_{t}$ is standard deviation and 2.33 is the $\mathrm{z}$ value of standard normal distribution (corresponding to the 99\% confidence interval selected).

And the VaR for $k$ days' period is estimated as:

$$
\operatorname{VaR}(k)=\sqrt{ } k \times 1 \text { day } \operatorname{VaR}
$$

This is referred to as the square root of time rule in VaR calculation. The square root of time rule implicitly assumes that daily returns are independent and identically distributed random variables. The variance-covariance method is suitable when the return distribution under analysis is either normal or very close to normal distribution. It is not able to capture fat-tailed behaviour if observed in the financial return series.

\subsection{Exponentially Weighted Moving Average (EWMA)}

It has been empirically observed that volatility tends to occur in clusters, which means that the periods of high volatility are followed by periods of higher volatility and periods of low volatility are followed by periods of lower volatility. This phenomenon can be referred to as "volatility is autocorrelated over time". However, in technical terms, it is known as the "volatility clustering" phenomenon. In this method for calculating volatility, more weight is given to the recent observations, and, as the observation goes back in the past, its importance for volatility calculation reduces. This mechanism captures volatility clustering very well. EWMA variance is computed as follows:

$$
\sigma_{t}^{2}=\lambda \cdot \sigma_{t-1}^{2}+(1-\lambda) \cdot \Delta r_{t}^{2}
$$

$\sigma_{t}^{2}=$ conditional variance,

$\lambda=$ decay factor,

$r_{t}^{2}=$ squared returns at time $t$. 
TheEWMA name derives from the fact that weights to past observations decline exponentially rather than linearly as in the standard deviation calculation. This technique is used by J. P. Morgan's Risk Metrics (1996) while calculating the volatility of financial asset returns. The closer the lambda $(\lambda)$ is to zero, the more weight is given to recent period squared return, and if the return series is highly volatile, then conditional variance will be highly volatile as well. Risk metrics forecasts of volatility are calculated using a constant $\lambda$ equal to 0.94 for daily data and equal to 0.97 for monthly data. These values are found to better capture the conditional volatility from their empirical research on financial returns series (Morgan (1996)). The study has also used $\lambda$ equal to 0.94 for calculating EWMA VaR. Finally, EWMA VaR is computed in a similar way as VcV VaR, only the volatility is calculated by Equation 3 above.

The method calculates the 1-day VaR with 99\% confidence level as:

EWMA VaR $=$ Amount of the position $\times 2.33 \sigma_{t}$,

where $\sigma_{t}$ is EWMA volatility and 2.33 is the $\mathrm{z}$ value of standard normal distribution (corresponding to the $99 \%$ confidence interval selected).

\subsection{Generalized Autoregressive Conditional Heteroskedasticity (GARCH)}

This is another important method of calculating volatility from past return series which captures volatility clustering phenomenon very well. This method assumes that there exists a "mean reversion" in long-term volatility and that over a sufficiently longer period of time volatility will tend towards its long-term equilibrium mean level. To check the presence of volatility clustering, the study has estimated various generalized autoregressive conditional heteroskedasticity (GARCH) models using the overall return series. A GARCH (p, q) model is specified as follows:

For a log return series $r_{t}$, let $a_{t}=r_{t}-\mu_{t}$, which is the mean corrected log return. Then, $a_{t}$ follows a GARCH (p, q) process if:

$$
\begin{gathered}
a_{t}=\sigma_{t} \varepsilon_{t,} \\
\sigma_{t}^{2}=\alpha_{0}+\sum_{i=1}^{p} \alpha_{i} a_{t-i}^{2}+\sum_{j=1}^{q} \beta_{j} \sigma_{t-j}^{2},
\end{gathered}
$$

where $\left\{\varepsilon_{\mathrm{t}}\right\}$ is a sequence of IID variable with zero mean and variance one. Then, $\alpha_{0}>0$, $\alpha_{i} \geq 0, \beta_{j} \geq 0$, and $\sum_{i=1}^{\max (p, q)}\left(\alpha_{\mathrm{i}}+\beta_{\mathrm{j}}\right)<1$. When $\alpha_{\mathrm{i}}>\mathrm{p}, \alpha_{\mathrm{i}}$ will be taken as zero, and so $\beta_{j}=0$ for $j>q$. The constraint on $\left(\alpha_{\mathrm{i}}+\beta_{\mathrm{j}}\right)$ implies that the unconditional variance of $a_{t}$ is finite, where $\sigma_{t}^{2}$ evolves over time as its conditional variance. 
To keep the GARCH model parsimonious and avoid overfitting, the study has analysed GARCH models with a maximum of four total lags, i.e. GARCH $(1,1)$, GARCH $(1,2), \operatorname{GARCH}(2,1)$, and $\operatorname{GARCH}(2,2)$ for the return series. The results are presented in Table 1.

Table 1. GARCH models estimate of Sensex return

\begin{tabular}{|c|c|c|c|c|c|}
\hline \multirow[b]{2}{*}{$\operatorname{GARCH}(\mathbf{p}, \mathbf{q})$} & \multicolumn{5}{|c|}{ Parameters } \\
\hline & $\alpha_{0}$ & $\alpha_{1}$ & $\alpha_{2}$ & $\beta_{1}$ & $\beta_{2}$ \\
\hline GARCH $(1,1)$ & $\begin{array}{c}1.74 \mathrm{E}-06 \\
(0.000)\end{array}$ & $\begin{array}{l}0.070015 \\
(0.000)\end{array}$ & & $\begin{array}{l}0.910085 \\
(0.000)\end{array}$ & \\
\hline GARCH $(1,2)$ & $\begin{array}{c}1.69 \mathrm{E}-06 \\
(0.000)\end{array}$ & $\begin{array}{c}0.778 \\
(0.000)\end{array}$ & & $\begin{array}{c}0.9452 \\
(0.000)\end{array}$ & $\begin{array}{l}-0.0326 \\
(0.8733)\end{array}$ \\
\hline GARCH $(2,1)$ & $\begin{array}{c}9.76 \mathrm{E}-06 \\
(1.000)\end{array}$ & $\begin{array}{c}0.0766 \\
(0.000)\end{array}$ & $\begin{array}{c}0.0043 \\
(0.8103)\end{array}$ & $\begin{array}{c}0.9090 \\
(0.000)\end{array}$ & \\
\hline GARCH $(2,2)$ & $\begin{array}{c}3.04 \mathrm{E}-06 \\
(0.0471)\end{array}$ & $\begin{array}{c}0.0742 \\
(0.000)\end{array}$ & $\begin{array}{c}0.0655 \\
(0.2469)\end{array}$ & $\begin{array}{c}0.1915 \\
(0.8104)\end{array}$ & $\begin{array}{c}0.6515 \\
(0.3704)\end{array}$ \\
\hline
\end{tabular}

Source: author's computation

Note: Values in the parentheses represent the respective p-values.

The parameter estimates show that none of the different orders of the GARCH model other than GARCH $(1,1)$ should be used for conditional volatility estimation as they are becoming insignificant (p-value is greater than $5 \%$ ). Overall, it has been observed from Sensex return series that volatility clustering is best captured by GARCH $(1,1)$. The stationary condition for GARCH $(1,1)$ is $\alpha_{1}+\beta_{1}<1$. For Sensex, the coefficients of GARCH $(1,1)$ are significant (i.e. $\alpha_{1}=0.070$ and $\beta_{1}=0.910$ ). As is typical of GARCH model estimates for financial asset return data, the sum of the coefficients of the lagged squared error and lagged conditional variance is generally found closer to unity $\left(\alpha_{1}+\beta_{1}\right.$ is 0.98$)$. However, as the sum is less than unity, the conditional variance equation tells us that we have "stationarity" in conditional variance. For stationary GARCH models, conditional variance forecast values will converge upon the long-term average value of variance as the prediction horizon increases. As the return series is best captured by GARCH $(1,1)$, the study has calculated volatility by using the form GARCH $(1,1)$. GARCH VaR is then computed by using the GARCH volatility and the parametric formula for VaR.

The method calculates the 1-day VaR with 99\% confidence level as:

$$
\text { GARCH VaR = Amount of the position } \times 2.33 \sigma_{t} \text {, }
$$

where $\sigma_{t}$ is GARCH volatility and 2.33 is the $\mathrm{z}$ value of standard normal distribution (corresponding to the $99 \%$ confidence interval selected). 


\subsection{The Historical Simulation Method}

This is the non-parametric method for calculating VaR. The main characteristic of simulation methods is that they use full valuation approach. This method does not assume that returns follow any particular distribution. The actual distribution of returns is generated from past data, and VaR is obtained from finding the relevant percentile (e.g. $1^{\text {st }}$ percentile for $99 \%$ VaR). It assumes that past data contain all the relevant information required to generate expected future returns. Hence, the quality of data becomes very important for this approach. Implicitly, it is assuming that the distribution of returns is not changing and is stationary over time.

\subsubsection{Steps for VaR Calculation}

In this method, the return series is generated over the past data window. The data series is then arranged in ascending order, having the return data start from the lowest and reach the highest point in the given period. VaR is then calculated by finding the required percentile as decided by the researcher.

The length of the past data is very important here as the number of days of past data should be sufficient to incorporate the tail of the return distribution. The data period should ideally capture economic scenarios which are expected in the near future. Also, if the number of past days from which market returns are generated is low, then the tail of the distribution may not be captured with sufficient accuracy. Empirically, the asset returns have moved to more extreme values in either side of the mean than what is suggested by the normal distribution curve (Venkatraman, 1997; Robert, 2009). This behaviour is known as leptokurtosis, wherein actual asset return distribution has thin waists and fat tails at the extremes (Figure 2).

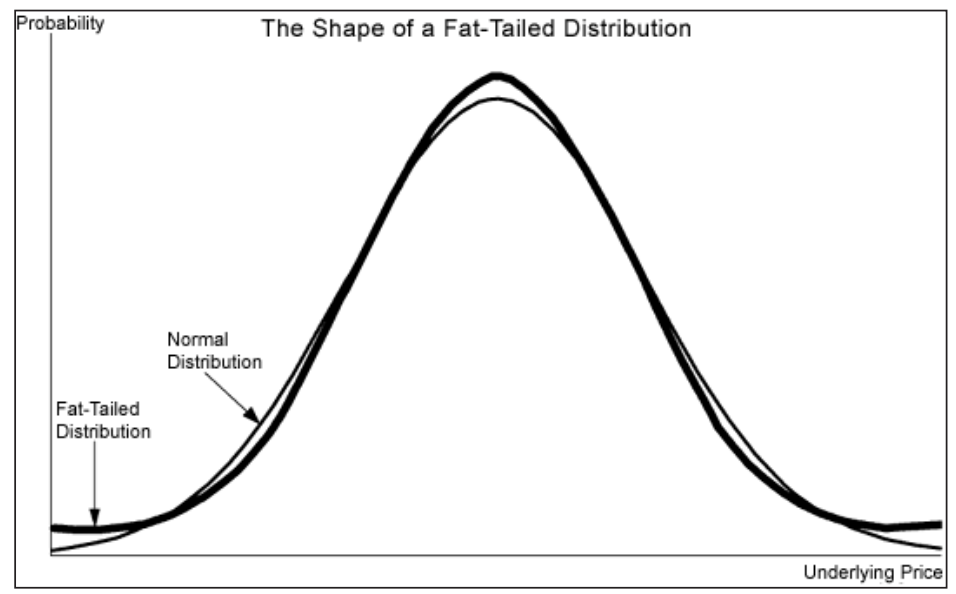

Figure 2. Fat tails of financial returns 
As Figure 2 above shows, if normal distribution is used as an approximation, then it would underestimate the probability of large extreme moves on either side of the distribution. As VaR is concerned with estimating extreme losses, it is very important that these fat tails are captured appropriately by the model. Empirically, it has also been observed that asset returns have reached much higher values on both sides of the mean of the normal distribution, which is not predicted well by the theory. Historical examples include the Black Monday (1987), the dot-com bubble (2001), and global financial crisis (2008). Historical simulation does not assume any distributional assumption. It is able to fully capture the fat-tailed behaviour of financial returns if they are contained in the past data, and it will accordingly show the VaR figure to reflect the true volatility of the asset under consideration.

\subsection{The Monte Carlo Simulation Method}

The basic concept of "Monte Carlo" is to repeatedly simulate a random process for the financial asset covering a wide range of possible situations. This method makes no distributional assumptions for return distribution, nor is the distribution generated by using past data. Here one assumes a suitable generating mechanism, and the data is generated by simulations through computer software applications. The designers of the risk measurement system are free to choose any distribution that they think appropriately describes the possible future changes in the market factors. Generally, if there is no significant market change expected, the beliefs about future market behaviour are based on the past behaviours observed. Therefore, designers of the system are free to choose any distribution that they think appropriately describes the past distribution. This approach is the most suitable one and, in most cases, the only option available to a researcher when the asset under analysis has non-linear payoff profiles such as options.

\subsubsection{VaR Estimation Procedure Steps}

a) A sequence of random numbers in the range from 0 to 1 is generated by using algorithms in a spreadsheet. The quantity depends upon the number of simulations needed to be performed. If it is believed that the algorithm process is not generating true random numbers, then the whole sequence of generated random numbers can be divided into various subparts, followed by taking the average of each subpart and then, finally, taking the sequence of these average numbers to further simulate the price path.

b) A distribution for generating the expected future natural log of asset price is assumed. The study assumes that the price $\left(\mathrm{p}_{\mathrm{t}}\right)$ follows a "random walk" process. This process is described as:

$$
\ln \left(p_{t}\right)=\ln \left(p_{t-1}\right)+u_{t}
$$


For generating the asset price, the study assumes that prices are continuous variables, and the asset price follows the geometric Brownian motion (GBM). This is the continuous counterpart of random walk process and is described as:

$$
d s=\mu_{t} s_{t} d t+\sigma_{t} s_{t} d z
$$

Here, $\mu_{t}$ is the expected rate of return on asset at time $t, s_{t}$ is asset value at time $t$, and $\sigma_{t}$ is the volatility of the stock price. The $d z$ random variable has the mean zero and variance $d t$, which inflicts price shock randomly to the portfolio value, and it does not depend on past information. In the above equation, two main parameters are very important to be determined: the expected return $(\mu)$ and the volatility of the underlying stock $\left(\sigma_{t}\right)$.

Determination of $\mu$ : The study assumes that if the asset belongs to the markettraded portfolio, it must bear the systematic risk arising from market movements. A rational investor will therefore always expect that the return on shares must be at least equal to the risk-free rate available in the market, the proxy of which can be taken as return on government securities, which are assumed to be risk-free in nature. Also, for taking additional risk, the investor must be expecting some risk premium over and above the risk-free interest rate. In theory, however, it is possible to eliminate the market risk completely by constructing a portfolio of shares and options. The famous "Black and Scholes option pricing model" used this approach when valuing options. This model values the options by assuming a risk-neutral portfolio. A risk-neutral portfolio is one that is not affected by market risk and gives the return independent of market risk movements. So, it is not totally unrealistic to assume that investors demand only the return equivalent to the risk-free rate of interest. The present study assumes that the expected return is simply the opportunity cost of capital, which is nothing but the risk-free rate of interest available elsewhere in the market. In the GBM equation, expected return is related linearly with time.

Determination of $\sigma_{t}$ : In the GBM, volatility is positively correlated to the time period over which it is estimated. It increases with the square root of time. Since for longer periods there are more expected variations in asset price, it is reasonable to assume that volatility increases with time. The square root formula for updating volatility is only applicable when the returns are uncorrelated over time.

c) Next, by using the inverse cumulative distribution function on the series of random numbers generated, the study obtains another series of observations of standard normal distribution having a mean of zero and a variance of one. This technique is called transformation method. This is required for modelling the GBM of asset prices.

d) Next, the asset prices are generated using the GBM formula and the stochastic terms generated above. Finally, different scenarios generated will lead to different 
values for the asset. The profit and loss distribution can then be formed, and VaR can be calculated using the appropriate percentile.

\subsubsection{Merits of the Monte Carlo Simulation Method}

The large number of scenarios generated provides a more reliable and a more comprehensive measure of risk than the analytic methods. Due to the various scenarios generated, this method captures well the fat-tailed behaviour of financial markets. As it assumes that prices are log-normally distributed, a significant amount of error compared to when we assume normal distribution is corrected. It is the only option which can handle very well non-linear payoffs that are associated with options. In many cases when we are dealing with complex exotic options, their risk can only be captured by Monte Carlo simulations and not by the other two methods mentioned earlier. It can incorporate different kinds of risks and is thus suitable for when the portfolio is exposed to various risks at the same time. It captures convexity of nonlinear instruments and updates the volatility when dealing with a longer time period. It can be used to simulate several alternative hypotheses about returns behaviour, which we assume to be such as white noise, autoregressive, etc.

\subsubsection{Limitations of the Monte Carlo Simulation Method}

The problem with this method is the computational effort required. When there is a very complex portfolio including a lot of options, the researcher will need to perform millions of simulation trials. It is both time- and energy-consuming. When the stochastic-process-generating mechanism does not match the actual return-generating mechanism, the final conclusions drawn would be misleading. This method also needs an assurance that the random numbers generated are truly random. If this is not assured, then the only way is to do simulations over a very large number of scenarios.

\section{Empirical Results}

The study uses daily Bombay Stock Exchange (BSE) Sensex index values as the benchmark for measuring equity market risk in India. The data extend over the period of 1 January 2006-31 December 2015, covering a time span of 10 years and a total of 2,480 observations. This range of 10 years of data allows us to draw meaningful insights from actual return distribution as the period is sufficiently long to study the behaviour of equity market. As per the required mandate for stress-testing framework from many regulatory bodies across the world (e.g. Comprehensive Capital Analysis and Review (CCAR) and Dodd Frank Annual Stress Testing (DFAST) in the USA and International Financial Reporting Standard (IFRS 9)), the modelling period must 
include at least one macro-economic slowdown period. The period undertaken includes the global financial crisis of 2008-2009. The modelling framework thus includes a macro-economic stress period, and the final model is suited for both baseline and stress macro-economic projections for a future time period.

The data is obtained from the official website of BSE. Continuously compounded returns are generated for the index as $R_{t}=\ln \left(I_{t} / I_{t-1}\right)$, where, $R_{t}$ is the return at time $t, I_{t}$ is the index value at time $t$, and $I_{t-1}$ is the index value at time $t-1$. In indicates the natural logarithm to base 'e'. The positive return $\left(R_{t}\right)$ indicates rising index value and is favourable while the negative return indicates losses and is unfavourable for the holder of the financial asset. The study uses continuously compounded returns. The percentage returns are not used as they are not symmetric in nature. Due to lack of symmetry, when the index value rises from the current value to a higher value, the calculated return will not be equal when the index value falls from the same increased value to the previous level.

Figure 3 shows the graphical plot of index returns. It is evident that the return series is highly volatile. The level of volatility is higher during the global financial meltdown of 2007-2009. The asymmetric behaviour in volatility is also observed. The volatility is much higher on the negative side (returns are reaching to $-9 \%$, whereas positive returns are reaching a maximum of close to $6 \%$ ). Regarding the normally made assumption for continuously compounded returns, it has also been observed that daily returns are on an average $0 \%$ while daily volatility is not, and it is generally found to be significant. This behaviour is observed as over the 10 years' period returns are more or less hovering around $0 \%$, but significant spikes in volatility are observed.

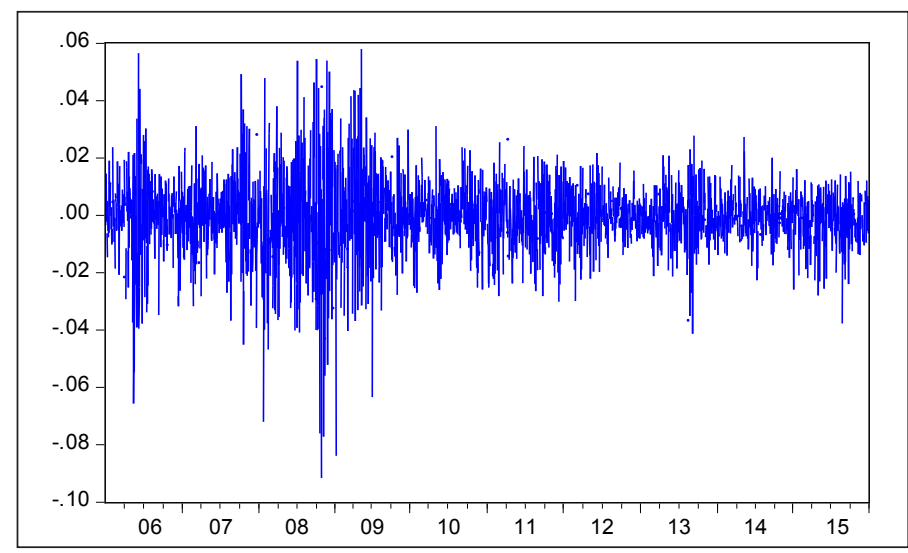

Source: author's generation

Figure 3. Sensex return plot for the period of 2006-2015

Table 2 below shows the descriptive statistics of Sensex returns. From the descriptive statistics, it becomes evident that the returns over this period are negatively skewed, 
with a skewness value of $-45 \%$. It is also observed that daily mean returns are close to $0 \%$, while daily standard deviation is found to be relatively significant $(1.34 \%)$. Fat-tailed behaviour in return series is also observed as the kurtosis value (7.29) is much higher than the value assumed for a normal distribution (3). It is evidencing that empirically Sensex returns have reached extremes on both positive and negative sides much more frequently than what is predicted by a normal distribution.

Table 2. Descriptive statistics of Sensex return

\begin{tabular}{lc}
\hline Metric & Sensex Return Series \\
\hline Mean & $-0.087 \%$ \\
\hline Median & $-0.065 \%$ \\
\hline Maximum & $5.799 \%$ \\
\hline Minimum & $-9.15 \%$ \\
\hline Std. Dev. & $1.34 \%$ \\
\hline Skewness & $-45.74 \%$ \\
\hline Kurtosis & 7.290 \\
\hline Total Observations & 2,480 \\
\hline & Source: author's computation
\end{tabular}

The study now attempts to examine the critical assumptions made under financial models since if these assumptions are not satisfied, the results generated by models may be inappropriate. If any assumption is found not to hold, then the study has tried to find what alternative distributional assumptions are best at describing the present data series.

Normality of the data series is a crucial assumption for parametric methods. In order to determine whether daily returns of Sensex are normally distributed or not, normality of the return distribution is tested by the Jarque-Bera (JB) test, the Anderson-Darling (AD) test, and the Kolmogorov-Smirnov test. The results are presented in Table 3 below.

Table 3. Normality tests of Sensex returns

\begin{tabular}{lc}
\hline Normality Tests & Sensex Return Series \\
\hline Jarque-Bera & $1995(0.0000)$ \\
\hline Anderson-Darling & $26.68(0.0000)$ \\
\hline Kolmogorov-Smirnov & $0.074(0.0000)$ \\
\hline
\end{tabular}

Source: author's computation

Note: Values in the parentheses represent the respective p-values. 
As the p-values of all three tests of normality are 0 , the null hypothesis of normality is rejected, and it can be strongly inferred that Sensex returns are nonnormal over the said period.

The study further uses graphical technique (Q-Q plot) to test for normality and the presence of heavy tails. The Q-Q plot of the returns is shown in Figure 4. It has steeper slopes at the tails, and the tails have their slope different from their central mass. These facts suggest that the empirical distribution of Sensex returns is not normal and has heavier tails than the reference normal distribution (Figure 4).

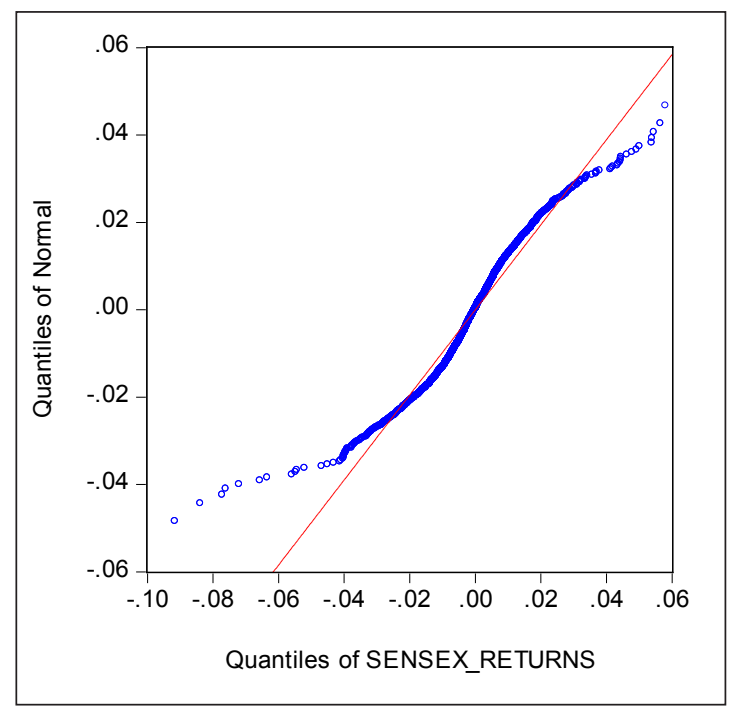

Source: author's generation

Figure 4. $Q-Q$ plots of Sensex return with normal distribution

To better capture the return distribution, the study further makes an attempt to see if the return distribution can be better approximated by other leptokurtic distributions which have higher kurtosis than the normal distribution. The t-distribution is a leptokurtic distribution having higher kurtosis than the normal distribution. Leptokurtic distributions are fat-tailed distributions having thicker tails, which are better able to capture the extreme moves of the distribution by providing a significant probability of exceeding from a given value in the tails of the distribution. As can be seen from Figure 5, the Q-Q plot of returns is better approximated by t-distribution than the normal distribution. The quantiles of Sensex returns are found closer to t-distribution quantiles, and hence it can be inferred that t-distribution is better able to capture the tails of the return series. 


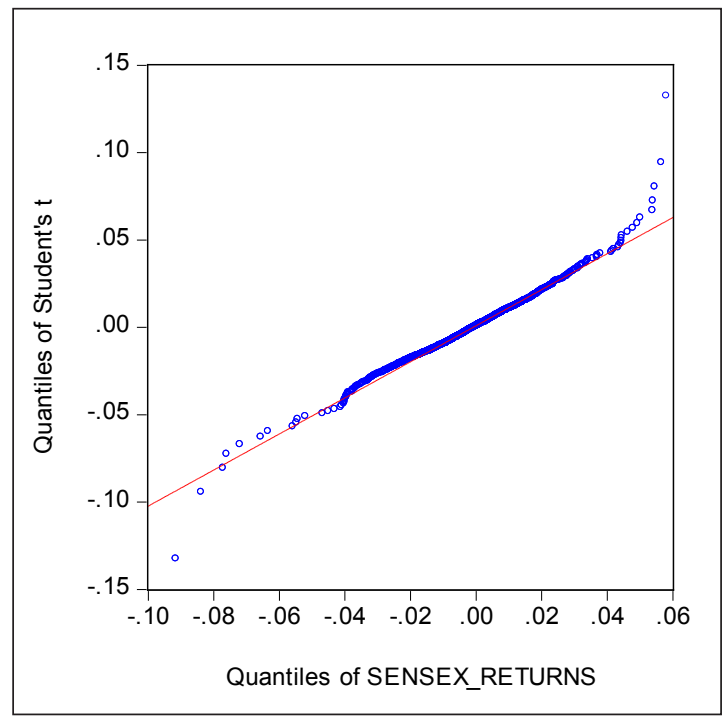

Source: author's generation

Figure 5. $Q-Q$ plots of Sensex return with student's t-distribution

The study further compares the actual distribution of returns with both normal and t-distributions (figures 6 and 7). The original empirical distribution of returns is plotted in Figure 6 with a superimposed normal distribution curve for making comparison. It is observed that actual returns are peaked at the mean value, thin at the waist, and thick at the bottom as the normal distribution, matching all the characteristics of fat-tailed behaviour. The actual returns series has further been compared with the more "leptokurtic" t-distribution in Figure 7. It shows that leptokurtic distribution such as "t" is better able to capture the actual empirical distribution of returns observed in the financial markets.

Overall, it can be inferred that Sensex returns are characterized by having thicker tails, and they are best captured by leptokurtic t-distribution, rather than the normal distribution.

Another very important assumption and requirement for financial return modelling is that the return series considered need to be stationary over the considered time period, otherwise the results may not be relied upon for future forecasting period. Stationarity of the variables is desirable and required whenever the past data is used to build models for future forecasting purposes. This is tested with the help of unit root test in Table 4 with the help of Augmented Dickey-Fuller "tau" statistics. This test checks for non-stationarity with three different functional forms: random walk, random walk with drift, and random walk with drift and trend. 


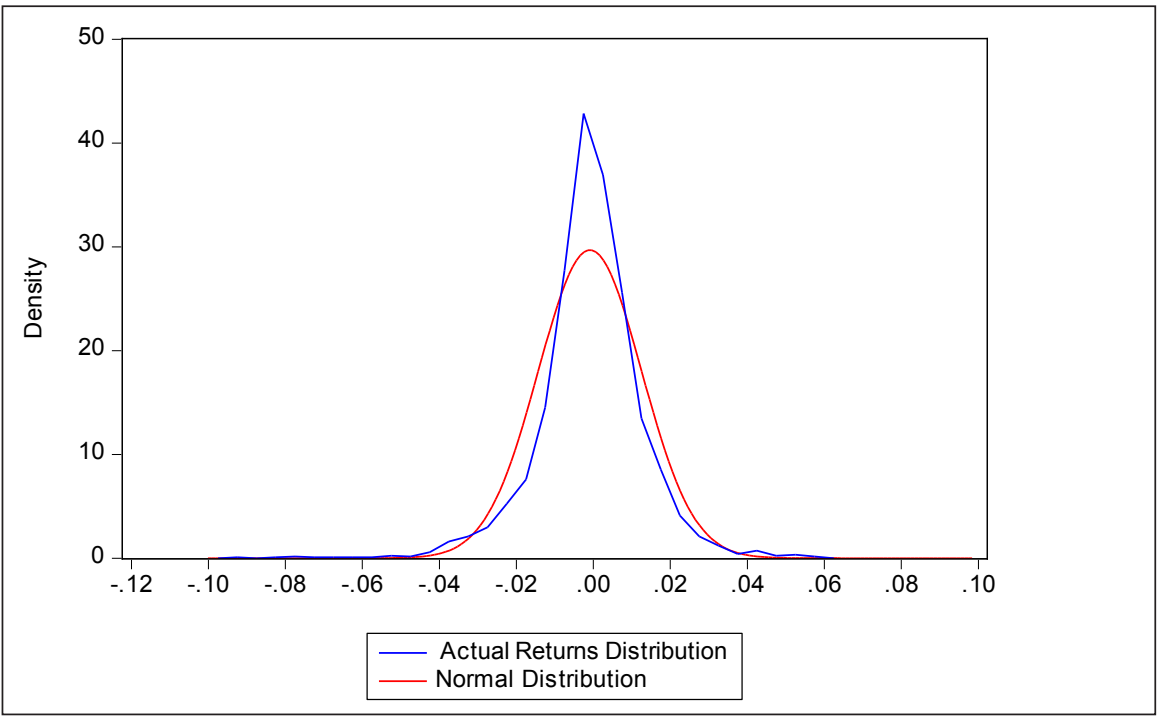

Source: author's generation

Figure 6. Actual return distribution compared with normal distribution

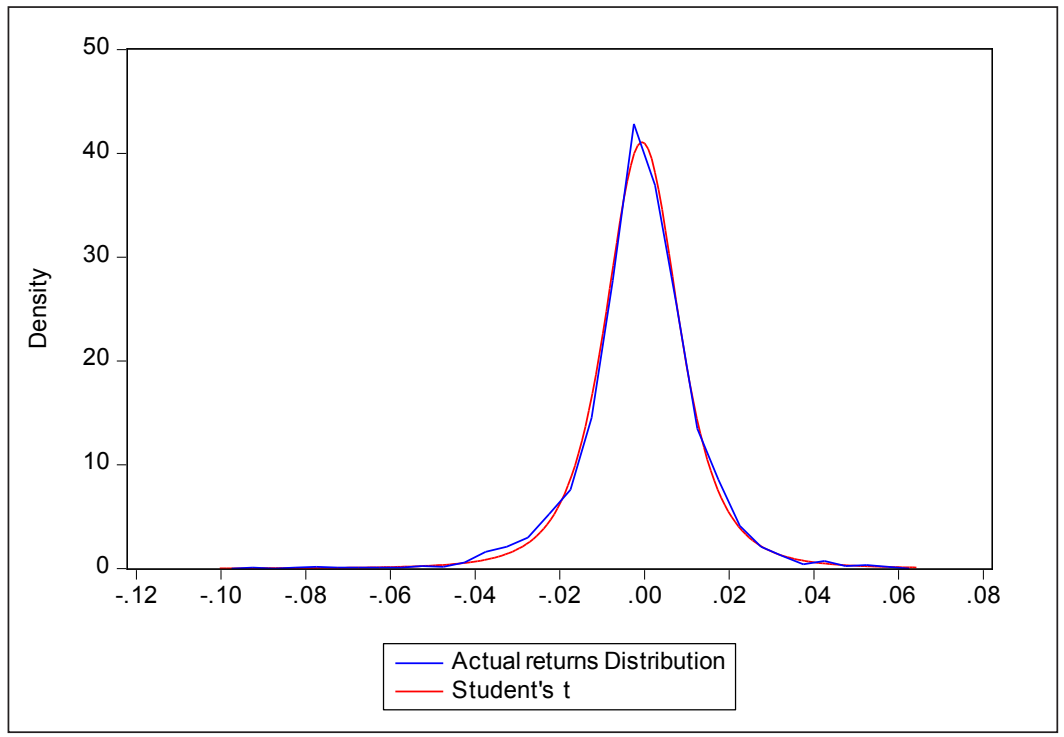

Source: author's generation

Figure 7. Actual return distribution compared with student's t-distribution 
Table 4. Checking the stationarity of Sensex returns

\begin{tabular}{lcccccc}
\hline \multicolumn{7}{c}{ Null Hypothesis: Sensex returns has a unit root } \\
\hline Model & $\begin{array}{c}\text { Trend } \\
\text { parameter }\end{array}$ & Drift & $\begin{array}{c}\text { Lagged } \\
\text { coefficient }\end{array}$ & $\begin{array}{c}\text { Tau- } \\
\text { statistics }\end{array}$ & $\begin{array}{c}\text { Critical } \\
\text { values } \\
\text { (5\% level) }\end{array}$ & H \\
\hline Random walk & NA & NA & -1.049 & -52.31 & -2.56 & 1 \\
\hline $\begin{array}{l}\text { Random walk with } \\
\text { drift }\end{array}$ & NA & -0.0009 & -1.054 & -52.53 & -2.86 & 1 \\
\hline $\begin{array}{l}\text { Random walk with } \\
\text { drift and trend }\end{array}$ & $-1.60 \mathrm{E}-07$ & -0.0007 & -1.0542 & -52.53 & -3.41 & 1 \\
\hline
\end{tabular}

Note: " $\mathrm{H}$ " stands for Boolean decision-making, where $\mathrm{H}=0$ means null hypothesis cannot be rejected. $\mathrm{H}=1$ means null hypothesis can be rejected.

The results of all three tests indicate that the null hypothesis of non-stationarity should be rejected, and it can be concluded that the return series is stationary, the behaviour shown by Sensex returns in the past is expected to continue in the future forecasting period as well, and any modelling exercise on the returns series can be statistically relied upon.

Table 5 below presents the results of VaR computation for Sensex using parametric (VcV, EWMA, GARCH) and non-parametric (HS and MCS) methods.

Table 5. VaR computation for Sensex

\begin{tabular}{lccccc}
\hline Method & VcV & EWMA & GARCH & HS & MCS \\
VaR & VaR & VaR & VaR & VaR \\
\hline $\begin{array}{l}\text { Value at Risk for } \\
\text { 1-day horizon (VaR) }\end{array}$ & $-3.219 \%$ & $-1.716 \%$ & $-1.581 \%$ & $-3.908 \%$ & $-1.661 \%$ \\
\hline & & & \multicolumn{3}{c}{ Source: author's computation }
\end{tabular}

Note: VaR figures are at $99 \%$ level of confidence with 1-day horizon.

It can be seen that the highest VaR figure is observed by using the historical simulation method $(-3.908 \%)$. This means that there is a $99 \%$ probability that on a typical trading day investors can expect that Sensex will fall in value by a maximum of $3.908 \%$ from its opening value, and there is only $5 \%$ probability that it can fall more than that. This information can help market participants to form appropriate market trading strategies and portfolio-management-related activities. VaR calculated with other parametric methods (VcV, EWMA, and GARCH) and nonparametric MCS predicts lower VaR estimates. Results suggest that Sensex returns have reached lower levels in the past which are not captured well by the normaldistribution-based parametric methods. This suggests that normal distribution assumption is not adequate for the Indian equity market, and it can be expected 
that the returns may very well deviate from the normal distribution in the near future. Overall, to be on the conservative side, it can be inferred that historical simulation is the best method for VaR estimation for the Indian equity market as represented here by Sensex. The study further tests this finding statistically in the next section of backtesting.

\section{VaR Backtesting}

The study finally tries to find the most appropriate VaR method which best captures the market risk arising from the Indian equity market (Sensex) by performing backtesting. Backtesting is a statistical process to ascertain the accuracy of the statistical model employed for prediction. This methodology compares the actual results with the results predicted by the model employed. If the actual loss on a given day exceeds the VaR predicted, then it means that the model is unable to predict accurately on that day, and it is considered as an actual exception. Exception is defined as a violation and indicates that the model employed is unable to predict the VaR accurately for the given day. The study uses 1-period ahead rolling window forecast to calculate the predicted number of exceptions. The study uses the first $500(1-500)$ data points from the beginning of the total historical time series data (1 Jan 2006 to 15 Jan 2008) to project VaR for the $501^{\text {st }}$ day (16 Jan 2008), then data from day 2 to 501 to project VaR for the $502^{\text {nd }}$ day, and so on. This process of updating VaR estimates continues up to 31 Dec 2015, and the total data points available to perform backtesting are 1980 (up to 31 Dec 2015). Finally, the actual and predicted exceptions are compared in the backtesting. The study uses 3 different tests to perform backtesting as mentioned below, and all of these tests use the total number of exceptions occurred as an input.

1. Actual number of exceptions test;

2. The Basic Frequency Backtest;

3. Proportion of Failure Likelihood Ratio (LR) test.

1) Actual Number of Exceptions: This test calculates the actual number of exceptions over the time period and compares them with the expected number of exceptions. For example, over a 100 days' time period and at $95 \%$ and $99 \%$ confidence levels, the expected number of exceptions are 5 and 1 respectively. This test only provides the direction that whether total exceptions are under- or overpredicted by the model employed. This test should be used in combination with the other two below-mentioned robust statistical methods to select the best VaR method.

2) The Basic Frequency Backtest: The basic frequency (or binomial) tests whether the observed frequency of tail losses (or frequency of losses that exceeded VaR) 
is consistent with the frequency of tail losses or the number of VaR violations ${ }^{1}$ predicted by the model. In particular, under the null hypothesis that the model is consistent with the data, the number of tail losses " $x$ " follows a binomial distribution. Given "n" return observations and a predicted frequency of tail losses equal to "p", this tells the probability of $x$ tail losses as:

$$
p_{r}(x \mid n, p)=\left(\begin{array}{l}
n \\
x
\end{array}\right) p^{x}(1-p)^{n-x},
$$

where " $\mathrm{n}$ " is the total number of observations, "p" is one minus confidence level, " $x$ " is the actual numbers of exceptions. The relationship between $x$ and binomial test probability value is that the probability value declines as $x$ gets bigger. When the number of observations increases, the binomial distribution can very well be approximated by a normal distribution. As the present study has a significant number of observations for backtesting (1980), it has employed the normal approximation test as given below:

$$
\mathrm{Z}=\frac{(x-n p)}{\sqrt{n \cdot p(1-p)}},
$$

where n.p is the expected number of exceptions and $[p .(1-p) . T]$ is their variance. This test follows the standard normal distribution with mean 0 and variance 1 . The test statistic uses left-tailed "z" statistic as VaR is concerned with predicting losses, and thus the left side of return distribution is used for calculating the number of exceptions. The null hypothesis assumes that the model is correctly calibrated, and if the value of the statistic is higher than the critical value of the $\mathrm{Z}$ distribution, then the null hypothesis is rejected and the model is recognized as being incorrectly calibrated and unable to predict VaR accurately.

3) Proportion of Failure LR Test: This is the likelihood ratio test suggested by Kupiec (1995) for backtesting, and it predicts whether there is a significant difference between actual and observed failure rate. Failure rate is defined here as the total number of exceptions $(x)$ observed over the total number of days (n). This test uses LR statistic as:

$$
L R=-2 \ln \left[\frac{p^{x}(1-p)^{(n-x)}}{\left(\frac{x}{n}\right)^{x}\left(1-\frac{x}{n}\right)^{(n-x)}}\right] \sim \quad \chi^{2}(1)
$$

1 Tail losses or VaR violation refers to the exceedance of actual VaR from the calculated VaR. Therefore, the number of VaR violation is the number of times it exceeds the calculated VaR. 
The LR statistic follows a chi-square distribution with 1 degree of freedom. The null hypothesis assumes that the model is correctly calibrated, and if the value of the statistic is higher than the critical value of the distribution, then the null hypothesis is rejected and the model is recognized as being incorrectly calibrated and unable to predict VaR accurately. Table 6 below provides the statistics obtained by using different VaR methods for 1-day holding period using 95\% confidence interval.

Table 6. VaR backtesting statistics at 95\% confidence interval

\begin{tabular}{lccccc}
\hline METHOD & $\begin{array}{c}\text { VCV } \\
\text { VaR }\end{array}$ & $\begin{array}{c}\text { EWMA } \\
\text { VaR }\end{array}$ & $\begin{array}{c}\text { GARCH } \\
\text { VAR }\end{array}$ & $\begin{array}{c}\text { HS } \\
\text { VaR }\end{array}$ & $\begin{array}{c}\text { MCS } \\
\text { VaR }\end{array}$ \\
\hline Actual number of exceptions & 98 & 111 & 160 & 96 & 114 \\
\hline $\begin{array}{l}\text { The Basic Frequency Backtest } \\
\text { z value }\end{array}$ & -0.103 & 1.237 & 6.289 & -0.309 & 1.546 \\
\hline $\begin{array}{l}\text { Proportion of Failure LR test } \\
\chi^{2} \text { values }\end{array}$ & 0.016 & 1.237 & 33.617 & 0.096 & 2.285 \\
\hline
\end{tabular}

Notes: expected number of exceptions $=99$ ( $5 \%$ of 1980); $\mathrm{z}$ critical value at $5 \%$ level $=1.645 ; \chi^{2}(1)$ critical value at $5 \%$ level $=3.841$

From the above table, it becomes evident that both statistical tests (the Basic Frequency Backtest and the LR test) find that VcV, EWMA, HS, and MCS are able to predict well the VaR as the null hypothesis cannot be rejected for all of them. From the actual number of exceptions, however, $\mathrm{VcV}$ and HS exceptions are very close to the expected number of exceptions (99). Hence, the study finds that at 95\% CI both VcV and HS are able to predict VaR reasonably well compared to other methods. However, at $95 \%$ confidence level, the tails of the distribution are not captured appropriately by the models; furthermore, the study observes tail behaviour by finding VaR at $99 \%$ confidence interval. Table 7 below provides the exceptions obtained by using different VaR methods for 1-day holding period using 99\% confidence interval.

Table 7. VaR backtesting statistics at 99\% confidence interval

\begin{tabular}{lccccc}
\hline METHOD & $\begin{array}{c}\text { VCV } \\
\text { VaR }\end{array}$ & $\begin{array}{c}\text { EWMA } \\
\text { VaR }\end{array}$ & $\begin{array}{c}\text { GARCH } \\
\text { VAR }\end{array}$ & $\begin{array}{c}\text { HS } \\
\text { VaR }\end{array}$ & $\begin{array}{c}\text { MCS } \\
\text { VaR }\end{array}$ \\
\hline Actual number of exceptions & 37 & 39 & 69 & 28 & 41 \\
\hline $\begin{array}{l}\text { The Basic Frequency Backtest } \\
\text { z value }\end{array}$ & 3.88 & 4.33 & 11.11 & 1.85 & 4.77 \\
\hline $\begin{array}{l}\text { Proportion of Failure LR test } \\
\chi^{2} \text { values }\end{array}$ & 12.01 & 14.66 & 75.12 & 3.03 & 17.51 \\
\hline
\end{tabular}

Note: expected number of exceptions = 20 ( $1 \%$ of 1980); $\mathrm{z}$ critical value at $1 \%$ level $=2.33 ; \chi^{2}(1)$ critical value at $1 \%$ level $=6.63$ 
From the above table, it becomes evident that from both statistical tests (the Basic Frequency Backtest and the LR test) only the historical simulation method is predicting well, and the null hypothesis cannot be rejected for both of the tests. None of the other methods is found to be predicting well, and the null hypothesis of the model being correct needs to be rejected for all other methods (VcV, EWMA, GARCH, and MCS). It is also observed from the actual number of exceptions that only historical simulation is predicting the exceptions closer to the expected. Hence, the study concludes that at $99 \%$ confidence interval only historical simulation is the best method for predicting VaR. Results also confirm the earlier observed phenomena of fat-tailed behaviour in the Indian equity market (Table 2). 99\% confidence interval is better able to capture the tail behaviour and can capture the variation in the tails of distribution as the historical simulation method does not make any distributional assumption and provides the VaR figure incorporating the fat-tailed behaviour.

Overall, combining the results of $95 \%$ and $99 \%$, the study concludes that historical simulation is the best predictive VaR model for the Indian equity market (Sensex).

\section{Summary and Conclusions}

The study shows the empirical findings related to the Indian equity market. It is observed that equity returns are non-normal, left-skewed, and characterized by excess kurtosis and exhibit fat-tailed behaviour. It is observed that "non-normal" Sensex return series is best captured by using "leptokurtic distributions", which allow for fat-tailed behaviour such as the t-distribution. It is further observed that return series is exhibiting the volatility clustering phenomenon. The return series is found to be stationary, and any statistical model can be relied upon for future forecasting period as well. The study supports the empirical findings of Venkatraman (1997), Nath and Samanta (2003), Sollis (2009), and Samanta et al. (2010). The findings proved to be different from those of Varma (1999), Chowdhury and Bhattacharya (2015), and Poornima and Reddy (2017). The study finds that the best method of VaR computation for the Indian equity market (Sensex) is historical simulation, which allows for non-normality, excess kurtosis, and the fat-tailed behaviour of financial returns. This finding is validated by three robust statistical backtesting methods. The backtesting is performed at $95 \%$ and $99 \%$ confidence intervals. At 95\%, results indicate that VcV, EWMA, HS, and MCS are able to predict well the VaR. For capturing the fat-tailed behaviour, the study further performs backtesting at 99\% confidence interval, where only HS stands as the best method for predicting VaR. Hence, the study concludes that the best method of VaR computation is historical simulation. 


\section{References}

Alexander, C. (2009). Market risk analysis, value at risk models. Vol. 4. John Wiley \& Sons.

Butler, C. (1999). Mastering value at risk: A step-by-step guide to understanding and applying VaR. Financial Times/Prentice Hall.

Chowdhury, P. D.; Bhattacharya, B. (2015). Estimation of value at risk (VaR) in the context of the global financial crisis of 2007-08: Application on selected sectors in India. Indian Journal of Research in Capital Markets 2(2): 7-25.

Coronado, M. (2000). Comparing different methods for estimating value-at-risk (VaR) for actual non-linear portfolios: Empirical evidence. Working paper Facultad de Ciencias Economicas y Empresariales, ICADE, Universidad P. Comillas de Madrid, Madrid, Spain.

Crouhy, M., Galai, D.; Mark, R. (2006). The essentials of risk management. Vol. 1. New York: McGraw-Hill.

Dowd, K. (2007). Measuring market risk. John Wiley \& Sons.

Dutta, D.; Bhattacharya, B. (2008). A bootstrapped historical simulation value at risk approach to S \& P CNX Nifty. The National Conference on Money and Banking, IGIDR, Mumbai, India.

Harmantzis, F. C.; Miao, L.; Chien, Y. (2006). Empirical study of value-at-risk and expected shortfall models with heavy tails. The Journal of Risk Finance 7(March): 117-135.

Kisacik, A. (2006). High volatility, heavy tails and extreme values in value at risk estimation. Institute of Applied Mathematics, Financial Mathematics/Life Insurance Option Program, Middle East Technical University - term project.

Kupiec, P. (1995). Techniques for verifying the accuracy of risk measurement models. The Journal of Derivatives 3(2): 76-84.

Maghyereh, A. I.; Al-Zoubi, H. A. (2006). Value-at-risk under extreme values: The relative performance in MENA emerging stock markets. International Journal of Managerial Finance 2(July): 154-172.

Morgan, J. P. (1996). Risk metrics technology document. Morgan Guaranty Trust Company of New York, New York. 35-65.

Nath, G. C.; Samanta, G. P. (2003). Value at risk: Concept and its implementation for Indian banking system. Available at: https://ssrn.com/abstract=473522.

Penza, P.; Bansal, V. K.; Bansal, V. K.; Bansal, V. K. (2001). Measuring market risk with value at risk. Vol. 17. John Wiley \& Sons.

Philippe, J. (2001). Value at risk: The new benchmark for managing financial risk. NY: McGraw-Hill Professional.

Poornima, B. G.; Reddy, Y. V. (2017). An analysis of portfolio VaR: Variancecovariance approach. IUP Journal of Applied Finance 23(3): 63-79. 
Şener, E.; Baronyan, S.; Mengütürk, L. A. (2012). Ranking the predictive performances of value-at-risk estimation methods. International Journal of Forecasting 28(4): 849-873.

Sollis, R. (2009). Value at risk: A critical overview. Journal of Financial Regulation and Compliance 17(November): 398-414.

Samanta, G. P.; Jana, P.; Hait, A.; Kumar, V. (2010). Measuring market risk - An application of value-at-risk to select government bonds in India. Reserve Bank of India Occasional Papers 31(1): 2-32.

Varma, J. R. (1999). Value at Risk Models in Indian Stock Market. Working paper no. 99-07-05. Indian institute of Management.

Venkataraman, S. (1997). Value at risk for a mixture of normal distributions: The use of quasi-Bayesian estimation techniques. Economic Perspectives - Federal Reserve Bank of Chicago 21: 2-13.

Zangari, P. (1996). An improved methodology for measuring VaR. RiskMetrics Monitor 2(1): 7-25. 